\title{
3C 273 AND DA 193 MAPPED WITH CRUSTAL DYNAMICS VLBI DA'TA
}

\author{
Patrick Charlot ${ }^{(1)}$, Jean-François Lestrade ${ }^{(2)}$, Claude Boucher ${ }^{(1)}$ \\ (1) Institut Géographique National \\ 2 Avenue Pasteur, F-94160, Saint-Mandé, France. \\ (2) Bureau des Longitudes \\ 77 Avenue Denfert-Rochereau, F-75014, Paris, France.
}

\begin{abstract}
Hybrid maps of 3C 273 and DA 193 at 2.3 and $8.4 \mathrm{GHz}$ have been produced with VLBI data acquired during a Crustal Dynamics campaign. By comparing to maps at previous epochs, superluminal components of $3 \mathrm{C} 273$ are clearly detected. DA 193 is relatively compact. Structure corrections for the VLBI delay used in astrometry have been estimated from these maps. Some corrections are significant when compared with the precision of models used in astrometry and geodesy.
\end{abstract}

The Crustal Dynamics Program (CDP) kindly provided us with the VLBI data acquired during the experiment conducted on North Pacific baselines on 1985 May 15 with a 6-station VLBI array (Mojave, Vandenberg, Hatcreek, Gilmore Creek, Kauai, Kashima). Figure 1 shows the u-v plane for $3 \mathrm{C} 273$ at $8.4 \mathrm{GHz}$. It is interesting that such coverage is more uniform than those provided by the other networks. Figure 2 shows the hybrid maps of DA 193 and 3C 273 made with these data.

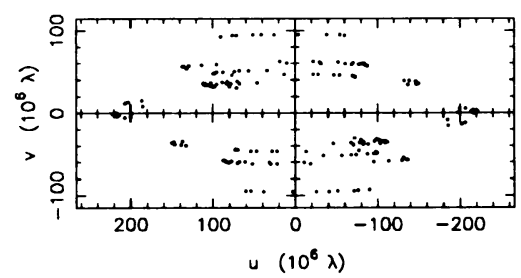

Figure 1. The u-v plane for $3 \mathrm{C} 273$ at $8.4 \mathrm{GHz}$.

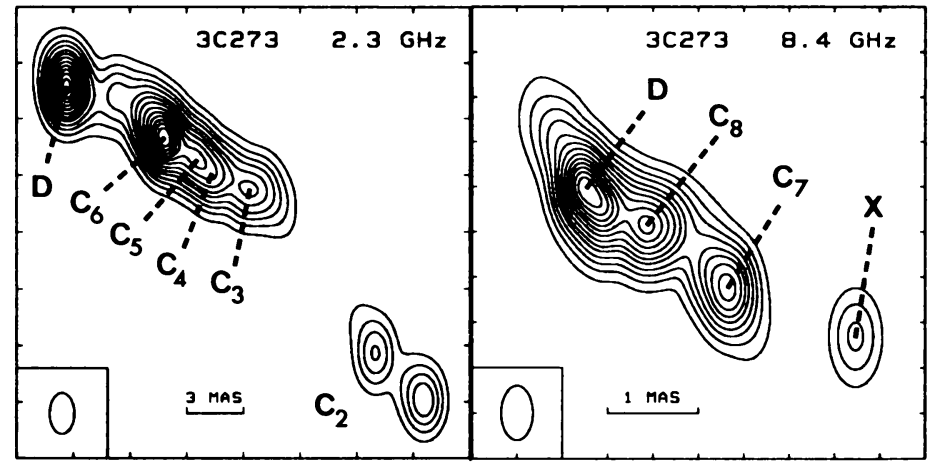

Figure 2. Hybrid maps of 3C 273 and DA 193 at 2.3 and $8.4 \mathrm{GHz}$. Contour levels: $1,3,6,10,16,22,30,40,50,60,70,80,90 \%$ of the peak brightness ( 1 and $3 \%$ not drawn for DA 193 at $8.4 \mathrm{GHz}$ ).

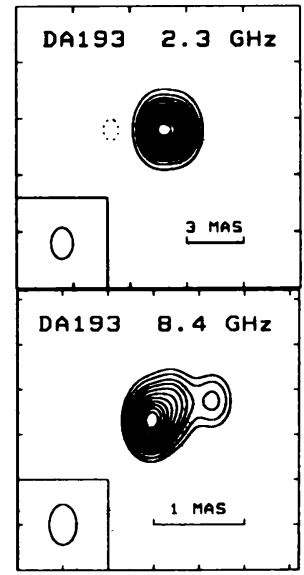

M. J. Reid and J. M. Moran (eds.), The Impact of VLBI on Astrophysics and Geophysics, 33-34. (C) 1988 by the IAU. 
Our maps of DA 193 are similar to the one found by Spangler et al (1983) at $5 \mathrm{GHz}$ at epoch 1981.7. However we find a slight extension in the $\mathrm{N}-\mathrm{W}$ direction that is not seen in their map. Our maps of $3 \mathrm{C} 273$ are consistent with previous maps published by Unwin et al (1985), hereafter U85, Biretta et al (1985), and Cohen et al (1987), hereafter C87. The separations and proper motions of the components of 3 C 273 are given by U85 and C87. We have used these values to predict the separations of the components at our epoch of observation (1985.37) in order to identify them. This comparison is summarized in Table I.

\begin{tabular}{|l|c|c|c|c|c|c|c|c|}
\multicolumn{1}{c|}{ Map at $2.3 \mathrm{GHz}$} & \multicolumn{3}{c|}{ Map at $8.4 \mathrm{GHz}$} \\
& $C_{2}$ & $C_{3}$ & $C_{4}$ & $C_{5}$ & $C_{6}$ & $X$ & $C_{7}$ & $C_{8}$ \\
\hline U85 or C87 & $20-25$ & 11.9 & 9.3 & 7.8 & & 3.6 & 2.1 & 0.7 \\
at 1985.37 & & \pm 0.5 & \pm 2.2 & \pm 0.2 & & \pm 0.2 & \pm 0.2 & \pm 0.2 \\
\hline Our maps & $21-26$ & 11.1 & 9.1 & 7.8 & 5.9 & 3.4 & 1.9 & 0.7 \\
\hline
\end{tabular}

Table I. Separations between the core $D$ and the components of $3 \mathrm{C} 273$ (milliarcseconds).

In our $2.3 \mathrm{GHz}$ map, the strongest component is assumed to be $C_{6}$. This component was first seen in 1981.10 at $10.7 \mathrm{GHz}$ (U85) when its separation from $D$ was 1.1 milliarcsecond. It is not seen in later maps at $10.7 \mathrm{GHz}(\mathrm{C} 87)$, but it is visible in our $2.3 \mathrm{GHz}$ map with a separation of 5.9 milliarcseconds from $D$. This implies a proper motion of 1.1 milliarcsecond per year. This proper motion is consistent with the measured proper motions of the other components. The weak emission region located between 21 and 26 milliarcseconds from the core may correspond to the fading component $C_{2}$.

The maps above can be used to derive astrometric corrections for the VLBI delays in order to refer the position of each source to a specific feature of its morphology (Thomas, 1980). Table II gives the magnitudes of these corrections at 2.3 and $8.4 \mathrm{GHz}$ in the cases of the complex source 3C 273 and of the simple source DA 193. These corrections must be included for modelling the VLBI delay at the 0.1 nanosecond level.

\begin{tabular}{|c|c|c|c|c|}
\multicolumn{1}{c}{} & \multicolumn{2}{c|}{ 3C 273} & \multicolumn{2}{c}{ DA 193} \\
\hline Maxi & $2.3 \mathrm{GHz}$ & $8.4 \mathrm{GHz}$ & $2.3 \mathrm{GHz}$ & $8.4 \mathrm{GHz}$ \\
Mean & $\mathbf{1 . 4 4}$ & 0.44 & $<0.01$ & 0.02 \\
RMS & 0.57 & 0.08 & $<0.01$ & 0.01 \\
& 0.57 & 0.15 & $<0.01$ & 0.01 \\
\hline
\end{tabular}

Table II. Structure corrections in the delays (nanoseconds) for $3 \mathrm{C} 273$ and DA 193 during the North Pacific CDP campaign on 1985 May 15.

\section{Acknowledgements:}

We are grateful to the Crustal Dynamics Program for providing us with the VLBI data analysed in this report. The Caltech VLBI package has been used to map the structures of the sources presented in this report.

\section{REFERENCES}

Biretta et al, 1985, Astrophys. J., 292: L5-L8.

Cohen et al, 1987, Astrophys. J., 315: L89-L92 (C87).

Spangler et al, 1983, Astrophys. J., 271: 44-50.

Thomas, J. B., 1980, NASA-JPL Publication 80-84.

Unwin et al, 1985, Astrophys. J., 289: 109-119 (U85). 